\title{
Dictynna
}

Dictynna

Revue de poétique latine

13 | 2016

Varia

\section{Il poeta costruisce la sua immagine: progettualità e autobiografia nel sermo oraziano}

\section{Mario Labate}

\section{OpenEdition}

\author{
Journals
}

Edizione digitale

URL: http://journals.openedition.org/dictynna/1314

DOI: 10.4000/dictynna.1314

ISSN: $1765-3142$

Notizia bibliografica digitale

Mario Labate, « II poeta costruisce la sua immagine: progettualità e autobiografia nel sermo oraziano », Dictynna [En ligne], 13 | 2016, mis en ligne le 20 décembre 2016, consulté le 10 septembre 2020. URL : http://journals.openedition.org/dictynna/1314 ; DOI : https://doi.org/10.4000/dictynna.1314

Questo documento è stato generato automaticamente il 10 settembre 2020.

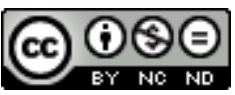

Les contenus des la revue Dictynna sont mis à disposition selon les termes de la Licence Creative Commons Attribution - Pas d'Utilisation Commerciale - Pas de Modification 4.0 International. 


\title{
Il poeta costruisce la sua immagine: progettualità e autobiografia nel sermo oraziano
}

\author{
Mario Labate
}

1 Nell'epistola ad Augusto Orazio delinea l'archeologia dello studium scribendi a Roma, quell'interesse per la letteratura che ha largamente soppiantato il tratto più caratteristico dell'antropologia e dell'ideologia romana, cioè l'inclinazione alle pratiche concrete della vita sociale, economica e politica. In questa celebre retrospettiva il poeta ricostruisce, sulla base delle ricerche dell'erudizione antiquaria ${ }^{1}$, il contesto agrario e rituale in cui si poteva immaginare il sorgere delle prime attività ludico-ricreative e artistico-letterarie: la festa del raccolto, il ristoro dalle dure fatiche dell'anno, le offerte alle divinità agresti e al Genio che governa la vita personale dell'individuo e dà soddisfazione a insopprimibili istanze vitalistiche e edonistiche:

Hor. epist. 2,1, 145-55

Fescennina per hunc invecta ${ }^{2}$ licentia morem

versibus alternis opprobria rustica fudit,

libertasque recurrentis accepta per annos

lusit amabiliter, donec iam saevos apertam

in rabiem coepit verti iocus et per honestas

ire domos inpune minax. doluere cruento

dente lacessiti; fuit intactis quoque cura

condicione super communi; quin etiam lex

poenaque lata, malo quae nollet carmine quemquam

describi: vertere modum, formidine fustis

ad bene dicendum delectandumque redacti.

"In queste usanze nacque la licenza dei fescennini, che in versi a botta e risposta profondeva rustici improperi e la libertà, ben accetta nella ricorrenza annuale, scherzò amabilmente, finché il gioco, fattosi crudele, cominciò a mutarsi in rabbia aperta e a portare impunemente minacce per le case degli onesti. Chi fu provocato dal morso sanguinoso si dolse e anche quelli che non erano toccati si preoccuparono della sorte comune e finì che fu proposta una legge e una pena, per 
impedire che uno fosse vittima di canzoni diffamatorie: cambiarono sistema, la paura del bastone li riportò a divertire senza parole d'insulto."

Il costume della festa contadina dà sfogo a pulsioni liberatorie e aggressive, connesse con rituali di fecondità animale e vegetale. Secondo questa ricostruzione oraziana, la primissima fase, quella identificata con la Fescennina licentia, consiste in uno scambio di opprobria rustica in versi: insulti, rozze battute aggressive di carattere contadino, secondo l'accezione sia denotativa che connotativa dell'aggettivo ${ }^{3}$. Il rituale rustico, recepito e in qualche modo 'regolarizzato' nell'ambito delle ricorrenze annuali, (notevole lo slittamento semantico da licentia a libertas) si evolve in un lusus piacevole e socialmente apprezzato (seconda fase: lusit amabiliter), fino a una terza fase, in cui il gioco (si noti ancora lo slittamento semantico lusus/iocus) si evolve ulteriormente verso l'aggressione violenta, che, con la copertura di impunità assicurata da un contesto originariamente rituale, tende a diventare una minaccia incontrollabile per la rispettabilità sociale (per honestas ire domos). Questa aggressività suscita non solo la reazione di coloro che soffrono in quanto vittime di un attacco gravemente lesivo (il morso che affonda nella carne fino a macchiare i denti di sangue), ma anche la reazione preventiva di chi, pur non essendo stato personalmente toccato, si sente vittima potenziale e si preoccupa del bene comune (condicione super communi) ${ }^{4}$.

2 Questi inconvenienti producono dunque contromisure di carattere legislativorepressivo $^{5}$ e le pene comminate a che i cittadini rispettabili non fossero vittima di canzoni diffamatorie furono efficaci: i Romani di quei tempi antichi cambiarono sistema e la paura del bastone li ricondusse a un divertimento che non ha bisogno di insulti e denigrazioni.

3 Per l'aggressività comico-rituale della preistoria agreste romana Orazio immagina un processo affine a quello che varie fonti greche, e Orazio stesso nell'Ars poetica ${ }^{6}$, presupponevano per lo sviluppo storico della commedia greca, dove è appunto il

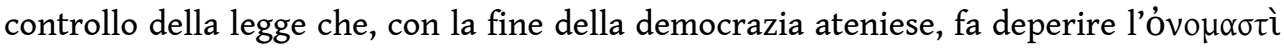
$\kappa \omega \mu \omega \delta \varepsilon \tilde{\imath} v$ in favore di forme comiche tendenzialmente meno aggressive e politicamente innocue ${ }^{7}$ :

Hor. ars poet. $281-4$

successit vetus his comoedia, non sine multa

laude; sed in vitium libertas excidit et vim

dignam lege regi: lex est accepta chorusque

turpiter obticuit sublato iure nocendi.

"Seguì a costoro [Tespi ed Eschilo] la commedia antica, con gran successo, ma la sua

licenza decadde in vizio e ingiuria: le occorreva la correzione della legge. Questa

venne sancita e, privo del diritto di offendere, avvilito il coro tacque".

La ricostruzione di questo percorso appartiene al poeta che assume la persona prestigiosa e autorevole del più grande poeta vivente di Roma, quello che ha titolo per 'insegnare ad Augusto' ${ }^{8}$ e per rivendicare al principe, e alla triade di poeti eccellenti cui egli stesso ha coscienza di appartenere, il merito di aver dato a Roma una letteratura all'altezza del prestigio della capitale imperiale ${ }^{9}$. Quella che Orazio sente di poter fare, come poeta di Roma, è una specie di autobiografia della poesia romana, che affonda le sue radici più in profondità rispetto all'inizio canonico determinato dall'incontro con la Grecia e dall'appropriazione creativa dei generi e dei capolavori (nonché degli standard qualitativi) della letteratura greca: Graecia capta ferum victorem cepit et artes / intulit agresti Latio.

Lo sguardo retrospettivo sulla preistoria della poesia romana autorizza e incoraggia un altro sguardo retrospettivo, che si concentri progressivamente prima sulla storia di un 
genere (quello che più ha a che fare con quelle lontanissime radici, come Orazio le ricostruisce: la satira), poi su una vera e propria autobiografia personale come poeta del sermo. ${ }^{10}$ Il lettore dell'Epistola ad Augusto è indotto cioè a rileggere, alla luce di questa ricostruzione, il percorso del genere satirico latino e il percorso di Orazio stesso come poeta della satira, responsabile di quelle riflessioni poetologiche che aveva praticato come connaturate al genere ${ }^{11} \mathrm{e}$ che possono ora presentarsi in una luce diversa, o forse possono rivelare con maggiore nitidezza il loro significato.

4 La satira 1, 4 s'impegnava a definire le coordinate del genere satirico di cui Lucilio era inventor e, al tempo stesso, la posizione di Orazio come successore di Lucilio e diverso interprete di quel genere di poesia ${ }^{12}$. Come tutti ricordano, il poeta esibisce la vocazione per una poesia che viene scritta per non essere letta, vocazione che viene messa in rapporto con la sua stessa natura personale, anzi con la sua stessa complessione psicofisica. Una paradossale autolimitazione, che viene messa in rapporto con la prevedibile diffidenza del pubblico per questo specifico genere di poesia:

Hor. serm. 1, 4, 17-24

di bene fecerunt, inopis me quodque pusilli

finxerunt animi, raro et perpauca loquentis

$\cdots$

cum mea nemo

scripta legat, volgo recitare timentis ob hanc rem,

quod sunt quos genus hoc minime iuvat

"Bene hanno fatto gli dèi, che m'hanno formato d'animo povero e piccino, di rare e scarse parole...mentre i miei scritti nessuno li legge, io che ho timore di recitare in pubblico; per questa ragione: che ci sono taluni cui questa poesia va pochissimo a genio..."

La radice della diffidenza, tanto radicale da tradursi in un sentimento di paura e di ostilità nei confronti della poesia tout court, viene ricondotta tuttavia non tanto a una specifica caratteristica del genere satirico quanto a una caratteristica del pubblico. Sono le manchevolezze morali del pubblico stesso, generalmente diffuse nella società, a produrre quella ipersensibilità che diventa allergia e rifiuto:

Hor. serm. 1, 4, 24-33

utpote pluris

culpari dignos. quemvis media elige turba:

aut ob avaritiam aut misera ambitione laborat.

hic nuptarum insanit amoribus, hic puerorum:

hunc capit argenti splendor; stupet Albius aere;

hic mutat merces surgente a sole ad eum, quo

vespertina tepet regio, quin per mala praeceps

fertur uti pulvis collectus turbine, nequid

summa deperdat metuens aut ampliet ut rem.

omnes hi metuunt versus, odere poetas.

"...giacché, per la maggior parte si meritano d'essere messi alla gogna. Scègline uno

a piacere dal mezzo del mucchio: o per avidità o per ambizione, poveretto,

s'affanna. Questo fa pazzie per amore delle donne sposate, dei ragazzini quest'altro; uno è rapito dal luccicare dell'argento; Albio istupidisce davanti a oggetti di bronzo; quest'altro scambia le sue merci dalle regioni del sole che sorge a quelle che intiepidisce al tramonto; e anzi si getta a capofitto attraverso i pericoli, come polvere ammassata nel turbine, per non perdere alcunché dal capitale o per accrescere il patrimonio. Tutti costoro temono i versi, hanno in odio i poeti."

Del resto, il modello della commedia greca arcaica, da cui Lucilio aveva mutuato il fondamento del suo ruolo di inventor della satira, connetteva necessariamente l'attacco personale a un giudizio morale negativo (che si presupponeva basato su valutazioni 
corrette) sui comportamenti e le scelte dei personaggi che erano bersagli dell'aggressione comica. L'óvo $\mu \alpha \sigma \tau \grave{~} \kappa \omega \mu \omega \delta \varepsilon \tilde{\imath} v$ attaccava chi si meritava di essere attaccato $^{13}$ :

Hor. serm. 1, 4, 1-6

Eupolis atque Cratinus Aristophanesque poetae

atque alii, quorum comoedia prisca virorum est,

siquis erat dignus describi, quod malus ac fur,

quod moechus foret aut sicarius aut alioqui

famosus, multa cum libertate notabant.

hinc omnis pendet Lucilius...

"Eupoli, Cratino e Aristofane, i tre poeti, e altri che furono gli autori della commedia antica, se c'era uno che meritava d'essere messo in berlina, perché furfante o ladro o adultero o sicario o altrimenti famigerato, lo bollavano senza tanti riguardi. Da qui Lucilio dipende tutto..."

L'avversario, cui la satira 1, 4 dà voce, controbatte proprio su questo punto, attribuendo invece al satirico una naturale propensione all'attacco indiscriminato, che non può non suscitare un allarme sociale generalizzato (longe fuge ${ }^{14}$. Come un incontrollabile animale aggressivo (è interessante che l'immagine del toro con il fieno sulle corna ci riporti al contesto agrario-contadino), il satirico è uno che, per far ridere, come il $\beta \omega \mu \mathrm{o} \lambda o ́ x o \zeta$ dell'Etica Nicomachea, non può fermarsi davanti a niente e davanti a nessuno, fosse anche un amico, fosse anche - paradossalmente - se stesso ${ }^{15}$ :

Hor. serm. 1, 4, 34-5

'faenum habet in cornu, longe fuge; dummodo risum

excutiat, sibi non, non cuiquam parcet amico...'

"Ha il fieno sulle corna; scappa lontano! pur di strappare una risata, non

risparmierà se stesso, né alcuno degli amici.."

La contestazione da parte del poeta di questa specifica accusa rivolta alla satira, non è immediata (il satirico sembra schivarla quasi con imbarazzo, soffermandosi a discutere sulla correttezza dell'identificazione tra satira e poesia), ma è il nocciolo della questione, cui è necessario tornare una volta conclusa la digressione:

Hor. serm. 1, 4, 64-5

nunc illud tantum quaeram, meritone tibi sit

suspectum genus hoc scribendi.

"Ora questo solo indagherò: se questo genere di letteratura ti sia sospetto a ragione."

Ha ragione o ha torto chi sospetta della satira? La satira merita sospetti e ostilità se chi in essa viene attaccato non merita di esserlo, viceversa non merita sospetti e rifiuti se i suoi bersagli meritano gli attacchi cui sono sottoposti. Questa specie di semplice sillogismo viene illustrato - satiricamente - con l'esempio di due accusatori di professione, Sulgio e Caprio, spauracchio dei latrones, ma del tutto innocui per chiunque conduca vita irreprensibile. A questa difesa del genere satirico Orazio sente il bisogno di associare un'ulteriore difesa del suo modo specifico di praticare il genere satirico stesso, ribadendo quell'autolimitazione che distingue definitivamente, nel suo caso, il satirico dall'accusatore di professione: la presunta rinuncia alla dimensione pubblica della comunicazione, il contenimento del pubblico della satira nello spazio ristretto di relazioni private e amicali:

Hor. serm. 1, 4, 65-73

Sulgius acer

ambulat et Caprius, rauci male cumque libellis, magnus uterque timor latronibus; at bene siquis et vivat puris manibus, contemnat utrumque. ut sis tu similis Caeli Birrique latronum, 
non ego sim Capri neque Sulgi: cur metuas me?

nulla taberna meos habeat neque pila libellos,

quis manus insudet volgi Hermogenisque Tigelli,

nec recito cuiquam nisi amicis idque coactus,

non ubivis coramve quibuslibet...

"Sulgio va in giro minaccioso e anche Caprio, terribilmente rauchi e con le scartoffie in mano, entrambi grande spauracchio per i malfattori; ma se uno vive onestamente e con le mani pulite, di entrambi non si dia alcun pensiero. Mettiamo pure che tu rassomigli a due furfanti come Celio e Birro, io però non somiglio né a Caprio né a Sulgio: perché dunque temermi? Nessuna bottega e colonnetta di libraio esponga i miei libretti, né sudi su di essi la mano del volgo e di Ermogene Tigellio; io non recito per nessuno, fuorché per gli amici, e soltanto forzato, non dovunque e davanti a chiunque..."

Ma la questione principale resta sempre la stessa e l'avversario non molla la presa. Il satirico viene identificato, e a sua volta denunciato, come uno che prova piacere a far male e che intenzionalmente dà sfogo a questa sua perversione, una specie di pericoloso sadismo sociale:

Hor. serm. 1, 4,78-9

'laedere gaudes'

inquit 'et hoc studio pravus facis.'

"'Ci godi a recare del danno' mi dice 'e lo fai di proposito, malvagio come sei'"

$\mathrm{E}^{\prime}$ a questo punto che un contesto specificamente autobiografico diventa fondamentale nella difesa e nella legittimazione del genere satirico ${ }^{16}$. La garanzia che l'aggressività del satirico non sia né possa diventare un fenomeno incontrollabile risiede fondamentalmente nel contesto delle sue relazioni personali, nella possibilità che possano essere chiamati, come testimoni a discarico, coloro con cui il satirico ha consuetudine di vita :

Hor. serm. 1, 4, 79-81

unde petitum

hoc in me iacis? est auctor quis denique eorum

vixi cum quibus?

"Dove sei andato a prenderla quest'accusa che mi scagli contro? Chi insomma te

l'ha imbeccata di quelli coi quali ho familiarità di vita?"

Un problema dibattuto nell'esegesi è l'attribuzione dei vv. 81-85 della satira 1, 4, che contengono l'identi-kit dell'"anima nera", cioè dell'individuo affetto da aggressività malevola e pericolosa, da cui la comunità civica ha il diritto-dovere di guardarsi (hic niger est, hunc tu Romane, caveto):

absentem qui rodit amicum,

qui non defendit alium culpante, solutos

qui captat risus hominum famamque dicacis,

fingere qui non visa potest, commissa tacere

qui nequit...

"Chi rode l'amico alle spalle, chi non lo difende quando un altro lo attacca, chi va in

caccia di risa sfrenate e della fama di uomo mordace, chi è capace di inventare ciò

che non ha visto, chi non sa tenere il silenzio su quello che gli è confidato..."

Io credo, con Kiessling Heinze e la maggior parte dei più importanti interpreti oraziani, che queste parole non appartengano all'adversarius, ma siano parte dell'autodifesa del satirico ${ }^{17}$. Lungi dall'assumere la difesa d'ufficio del diritto di laedere, il satirico quasi rovescia la prospettiva, denunciando lui stesso, con enfasi indignata, i pericoli di un discorso teso a far ridere e a conquistare fama di dicacitas. Il niger homo è quello che attacca gli amici, che non li difende quando sono attaccati dagli altri: la misura che permette di stabilire, senza incertezze, se la poesia che critica e attacca sia o non sia, 
effettivamente, un pericolo sociale risiede all'interno del rapporto di amicizia. Se l'aggressività fosse infatti introdotta e accolta nelle relazioni amicali, allora sarebbe giusto pensare che ad essa non può essere posto alcun limite, e la società tutta (anche quelli che, per ora, non sono oggetto di attacco) avrebbe ragione di sentirsi minacciata. Un atteggiamento di questo genere nei confronti di un amico non è solo qualcosa che il satirico sente di dover escludere come proprio del soggetto della satira, ma è un comportamento che anzi egli vuole mettere sotto accusa, facendone oggetto di satira, nel memorabile sketch in cui l'adversarius è immaginato come l'amico malevolo che, nel far finta di difendere Petillio Capitolino, ne approfitta per colpirlo perfidamente con le sue malignità.

7 La discussione diatribica contro il rigorismo stoico e a favore dell'indulgenza nell'amicizia, che è il tema della satira 1,3 , aveva del resto sgomberato il campo da questo pericolo: con gli amici, una volta stabilito quel giudizio complessivamente positivo che è il presupposto del rapporto di amicizia stesso, bisogna saper rinunciare alla critica e anzi attenuarne il più possibile i difetti prendendoli in bonam partem ${ }^{18}$. Naturalmente il presupposto fondamentale risiede nella correttezza del giudizio complessivo:

Hor. serm. 1, 3, 69-72

amicus dulcis, ut aequum est, cum mea conpenset vitiis bona, pluribus hisce, si modo plura mihi bona sunt, inclinet, amari si volet: hac lege in trutina ponetur eadem. "L'amico mio dolce, secondo equità, pesi assieme ai difetti i miei pregi, e più verso questi, che sono più numerosi, se pure in me i pregi sono più numerosi, egli faccia pendere il piatto, se vorrà essere amato: a questa condizione sarà posto nella stessa bilancia"

8 La teorizzazione sull'amicizia aveva molto insistito sul delicatissimo problema della scelta degli amici. Nel trattato de amicitia di Cicerone, Lelio attribuisce a Scipione la più viva preoccupazione e la più netta condanna dell'avventatezza e della superficialità in una materia tanto delicata:

Cic. Lael. 62

sed (saepe enim redeo ad Scipionem, cuius omnis sermo erat de amicitia) querebatur, quod omnibus in rebus homines diligentiores essent; capras et oves quot quisque haberet, dicere posse, amicos quot haberet, non posse dicere, et in illis quidem parandis adhibere curam, in amicis eligendis neglegentis esse nec habere quasi signa quaedam et notas, quibus eos, qui ad amicitias essent idonei, iudicarent.

"[Scipione] si lamentava del fatto che in tutte le cose gli uomini fossero più diligenti; quante capre o quante pecore avesse, ciascuno era in grado di dirlo; quanti amici, no; e che nell'acquistare quegli animali adottassero ogni attenzione, nello scegliere gli amici fossero invece negligenti e non avessero come dei segni e degli indizi, in base ai quali giudicare chi fosse degno di amicizia"

Lael. 78

omnino omnium horum vitiorum atque incommodorum una cautio est atque una provisio, ut ne nimis cito diligere incipiant neve non dignos.

"Insomma, da tutti questi difetti e inconvenienti c'è un solo modo di cautelarsi e premunirsi: non cominciare troppo presto a voler bene, non voler bene a chi non ne è degno. "

La garanzia, da questo punto di vista, per quello che riguarda il contesto amicale in cui Orazio colloca la sua legittimazione come poeta della satira è nella scena 
'autobiografica' del primo incontro con Mecenate, paucorum hominum et mentis bene sanae (Hor. serm. 1, 9, 44) ${ }^{19}$ :

Hor. serm. 1, 6, 56-64

ut veni coram, singultim pauca locutus -

infans namque pudor prohibebat plura profari -

non ego me claro natum patre, non ego circum

me Satureiano vectari rura caballo,

sed quod eram narro. respondes, ut tuus est mos,

pauca; abeo, et revocas nono post mense iubesque

esse in amicorum numero. magnum hoc ego duco,

quod placui tibi, qui turpi secernis honestum

non patre praeclaro, sed vita et pectore puro.

"Quando ti venni davanti, poche cose io dissi, balbettando (la soggezione mi legava

la lingua, mi impediva di dire di più): non che ero nato da padre illustre, non che mi

facevo portare in giro per le mie terre da un cavallo tarentino, ma quello che ero ti

dico. Mi rispondi, com'è tuo costume, poche parole; vado via e tu mi richiami nove

mesi più tardi e m'inviti a essere nel numero dei tuoi amici. Io la ritengo una cosa

grande questa, essere piaciuto a te, che sai distinguere l'uomo onesto dall'indegno,

non per la nobiltà di suo padre, ma per purezza di vita e di cuore. "

Testimonianze di carattere autobiografico escludono dunque l'eventualità che la satira diventi un 'pericolo pubblico' liberando indiscriminatamente le sue potenzialità aggressive. Di più: il carattere socialmente accettabile della satira non è dato una volta per tutte. La satira è un'arma ed è quindi, sempre, almeno potenzialmente pericolosa. Spetta di volta in volta al satirico dimostrare che tutte le cautele sono state adottate, che non c'è ragione di nutrire sospetti o di reagire con contromisure più o meno radicali. Ma le garanzie che il satirico deve sentirsi tenuto a dare non sono 'generiche' (nel senso del genere letterario), ma sono di carattere strettamente personale, sono legate alla persona e alla vita del poeta. E' questo il valore poetologico del ricondurre il gesto satirico alla più personale delle rievocazioni autobiografiche, l'educazione paterna:

Hor. serm. 1, 4, 105-6

insuevit pater optimus hoc me

ut fugerem exemplis vitiorum quaeque notando.

"quel galantuomo di mio padre me l'ha insegnato, a fuggire i vizi facendomeli

conoscere uno ad uno con degli esempi."

La definizione della satira come autoeducazione, come naturale prosecuzione di quel modello educativo empirico tutt'altro che estraneo alla tradizione filosofica greca, ma anche compatibile con il buon senso del tradizionalismo romano ${ }^{20}$, può essere esibita come la migliore garanzia che libertas e iocus si manterranno entro limiti socialmente accettabili:

Hor. serm. 1, 4, 100-5

hic nigrae sucus lolliginis, haec est aerugo mera; quod vitium procul afore chartis, atque animo prius, ut siquid promittere de me possum aliud vere, promitto. liberius si

dixero quid, si forte iocosius, hoc mihi iuris cum venia dabis...

"Questo sì è nero sugo di seppia, questa è ruggine bella e buona; questo vizio sarà lontano dalle mie pagine e, prima ancora, dall'animo mio: se una promessa sincera su di me posso fare, io lo prometto. Se mi accadrà di dire qualche cosa con un certa franchezza, o motteggiando un tantino, questo diritto me lo concederai e me ne darai licenza..." 
La satira 2,1 dimostra che la questione delle regole del genere e della sua 'accettabilità sociale' è strutturale, e non può dunque ritenersi risolta una volta per tutte. Il proemio del secondo libro è un componimento difficile, che sembra resistere a numerosi e intelligenti tentativi di decifrarne l'ambiguità e di rintracciarvi un compiuto percorso di senso ${ }^{21}$.

Tutti probabilmente sottoscriverebbero la semplice constatazione che si mette qui in scena una discussione sul genere satirico e i suoi interpreti (in buona sostanza Lucilio e Orazio), che riguarda ambedue gli elementi riconosciuti come assi portanti del primo libro delle Satire oraziane: l'aggressività e l'autobiografia. Ma se si cerca di andare oltre, se si cerca di capire, al di là delle schermaglie dialettiche tra Orazio e Trebazio, il nesso logico profondo tra questi due elementi e quindi il valore poetologico che il lettore è chiamato a ricavare dal testo, le cose si fanno complicate, controverse.

Nell'ambito di questa comunicazione, mi propongo un tentativo modesto (o forse troppo ambizioso?): ricostruire quella che la lezione della filologia tedesca ci insegnava essere una sorta di necessaria operazione preliminare, seguire il Gedankengang, la concatenazione del pensiero, i nessi espliciti (o più volte impliciti) per cui un pensiero, un argomento, un'immagine si collega, si sviluppa o si contrappone, magari ellitticamente, a ciò precede e a ciò che segue (Gian Biagio Conte la esaltava nel metodo di un suo maestro, Friedrich Klingner ${ }^{22}$, ma il metodo apparteneva con accentuazioni diverse, a vari grandi interpreti di poesia augustea: Heinze, Rothstein etc.).

11 Come tutti ricordano, all'amico giureconsulto Trebazio Testa viene chiesto un parere su come Orazio debba regolarsi di fronte a opposte critiche che anonimi contestatori rivolgono al suo modo di interpretare il ruolo del satirico. Da una parte l'accusa è di ultra legem tendere opus: sembrerebbe di capire che gli si rimprovera di usare ferocemente la satira come un'arma (tendere fa pensare all'arco armato, pronto a scoccare le frecce), al di là di quanto consenta la legge (in prima istanza la legge come "norma giuridica"); dall'altra parte, tutto quello che Orazio scrive è giudicato sine nervis, privo di energia e della tensione aggressiva ed espressiva ${ }^{23}$ connaturata al genere (contrariamente alla lex operis, il poeta è fiacco e l'arco è disarmato). Il dilemma sembra essere dunque tra una satira aggressiva, coerente con la lex operis stabilita da Lucilio, ma esposta alle proteste di vittime reali o potenziali, pronte ad appellarsi ai rigori della norma giuridica; e una satira fiacca ed inerme, che i fautores Lucilii si sentono di condannare come violazione delle norme del genere, ritorcendo anzi su Orazio, sul piano degli animi piuttosto che su quello dello stile, le critiche di abbondante facilità che egli rivolto all'inventor:

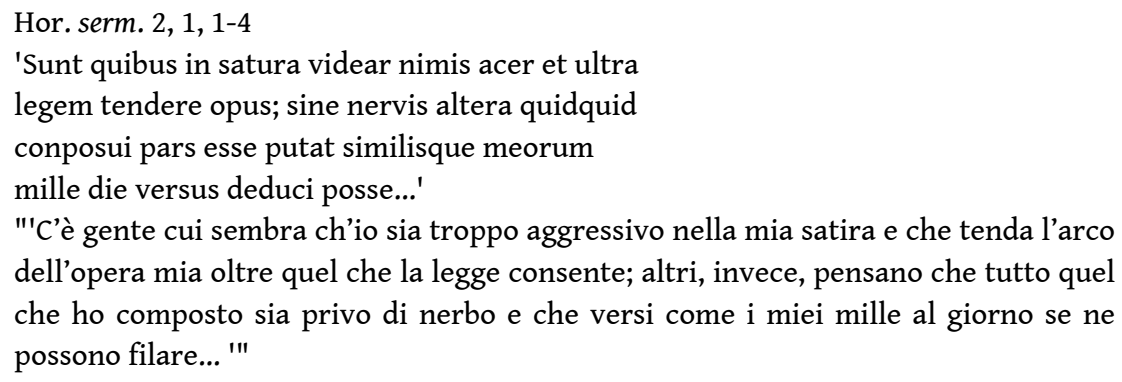

12 Una prima sorpresa è che il satirico rinunci a presentare la questione come immediatamente chiusa, approfittando della facile via d'uscita assicurata da due critiche opposte che, secondo una elementare dialettica, potrebbero apparire vicendevolmente smentite. E invece la soluzione che il satirico fa proporre al suo 
interlocutore (quiescas) suggerisce che il problema è aperto, che è difficile, e che addirittura la soluzione migliore sarebbe di sottrarsi gordianamente al dilemma rinunciando del tutto non soltanto alla satira, ma anche alla poesia tout court:

Hor. serm. 2,1, 4-6

'Trebati

quid faciam? praescribe.' 'quiescas.' 'ne faciam, inquis,

omnino versus?' 'aio.'

"'Trebazio, prescrivimi tu cosa fare'. 'Stattene quieto'. 'Non fare più versi per

niente, è questo che dici?' 'Questo è il responso'. "

La replica che motiva il rifiuto di un parere tanto drastico quanto risolutivo adduce anzitutto pretestuose ragioni di personale necessità, che si fondano sulla stessa complessione psico-fisica del poeta (verum nequeo dormire). L'elucubratio poetica sarebbe un rimedio per l'insonnia o comunque un modo per occupare ore notturne di veglia involontaria: un'affermazione ironica, piena di understatement (dunque adatta alla satira) della serietà della poesia come bisogno insopprimibile ${ }^{24}$. Dimostrata in qualche modo la 'necessità' della poesia, resta ancora da dimostrare le necessità di quella poesia (la satira), Quanto ai generi maggiori (l'epica), si ricorre facilmente alle scuse d'incapacità che sono di casa nelle recusationes e si può dare cosi per dimostrata la necessità di un genere minore come quello inventato e praticato da Lucilio. Più imbarazzante scartare l'ipotesi, prospettata dall'amico giurista, secondo cui lo spazio della satira potrebbe opportunamente ospitare, sull'esempio di Lucilio, un'opzione diversa, quella di una poesia morale di carattere più celebrativo che aggressivo, intesa $\mathrm{a}$ mettere in luce le virtù degli amici anziché i vizi dei nemici $(2,1,16-7)$. Orazio se la cava con un'acrobazia, allontanando (per lo meno nel tempo) l'elogio dell'amico attraverso un elogio più grande del più grande degli amici: Scipione aveva probabilmente gradito gli elogi dell'amico Lucilio, ma Cesare si mostra consapevole che mostrarsi renitente all'elogio (e alla possibile adulazione) è prova di grandezza ancora maggiore.

13 Ecco dunque che la discussione è tornata, più o meno, al punto di partenza: la satira come strumento di aggressione. Ma con una differenza. Dal punto di vista del giurista, stabilire se Orazio, come satirico, sia troppo o troppo poco aggressivo non è particolarmente rilevante. E' la satira stessa come aggressione che costituisce il problema. L'argomento è lo stesso dell'adversarius di 1, 4, l'allarme sociale suscitato dall'attacco alle persone ${ }^{25}$ :

Hor. serm 2, 1, 21-3

'quanto rectius hoc quam tristi laedere versu

Pantolabum scurram Nomentanumque nepotem,

cum sibi quisque timet, quamquam est intactus, et odit.'

"'Quanto meglio questo, che aggredire con versi maligni Pantolabo il buffone e

Nomentano lo scioperato: ecco che ognuno allora, anche se non viene toccato, teme

per sé stesso e prende ad odiarti'. "

La replica ribadisce l'argomento della necessità personale (il poeta non può che fare poesia e non può che fare quella poesia), spostando tuttavia l'accento dalla fisiopatologia alle inclinazioni naturali del carattere. Ciascun individuo ha il suo studium, diverso da quello di tutti gli altri e ad esso non può sfuggire. Fare poesia alla maniera di Lucilio è uno di questi, ed è quello che è toccato ad Orazio:

Hor. serm. 2, 1, 27-9

quot capitum vivunt, totidem studiorum

milia: me pedibus delectat claudere verba

Lucili ritu 
"quante migliaia di teste, tante migliaia di inclinazioni: io mi diletto di chiudere le parole nel verso, alla maniera di Lucilio..."

E' a questo punto che il filo del Gedankengang sembra quasi spezzarsi e la concatenazione logica, che nel sermo segue un percorso non sempre rettilineo, anzi prevede costituzionalmente slittamenti, deviazioni, cambiamenti di rotta, si rende poco visibile, si inabissa (magari come un fiume carsico, per riapparire più avanti). Ma dovere dell'interprete è cercar di seguire anche il tratto sotterraneo del percorso, seppure l'operazione comporti un'assunzione di responsabilità e anche qualche rischio.

Che cosa vuol dire Lucili ritu? Viste le premesse del dilemma iniziale, ci si aspetterebbe una definizione della lex operis sul terreno dell'aggressività, e invece sentiamo parlare della satira luciliana come luogo dell'autobiografia. I libri sono dei sodales cui il poeta confida i suoi segreti in ogni circostanza, positiva o negativa, della sua vita, cosicché la sua lunga vita stessa vi risulta raffigurata come su una votiva tabella:

Hor. serm. 2, 1, 30-3

ille velut fidis arcana sodalibus olim

credebat libris neque, si male cesserat, usquam

decurrens alio neque, si bene; quo fit ut omnis

votiva pateat veluti descripta tabella vita senis.

"Come a fedeli compagni, ai libri egli soleva affidare i suoi segreti, né altrove ricorreva se le cose gli andavano male, né se gli andavano bene: perciò avviene che tutta la vita di questo vecchio ci sta davanti agli occhi, come fosse dipinta su un quadretto votivo."

Che rapporto logico viene istituito - se ne viene istituito uno - tra aggressività e autobiografia? Per di più: quando Orazio, immediatamente dopo, passa a illustrare e a definire il suo modo di essere seguace di Lucilio (sequor hunc) non parla di libri come una specie di diario personale, ma dà piuttosto informazioni (come in una sphragis) sulla sua terra d'origine, soffermandosi sulle caratteristiche storiche e geo-politiche della colonia di Venosa ${ }^{26}$ :

Hor. serm. 2, 1, 34-9

sequor hunc, Lucanus an Apulus anceps;

nam Venusinus arat finem sub utrumque colonus,

missus ad hoc pulsis, vetus est ut fama, Sabellis,

quo ne per vacuum Romano incurreret hostis,

sive quod Apula gens seu quod Lucania bellum

incuteret violenta.

"Seguo il suo esempio io, non si sa se Lucano o Apulo: infatti il colono di Venosa ara

i campi a cavaliere tra le due regioni, inviato qui, raccontano antiche storie, dopo

che ne furono scacciati i Sabelli, per questo scopo, che i nemici non potessero

piombare addosso ai Romani attraverso un territorio disabitato, fosse la gente

d'Apulia o la Lucania bellicosa a scatenare la guerra. "

E' per questa via che il fiume carsico riaffiora in superficie. Essere seguace di Lucilio è messo in relazione con il più autobiografico degli eventi, il fatto di essere nato a Venosa, colonia militare stabilita come avamposto difensivo del territorio di Roma, un presidio in grado di opporre resistenza armata ad attacchi di popolazioni bellicose ${ }^{27}$. Chi nasce a Venosa ha una specie di vocazione naturale all'uso delle armi, ma in funzione eminentemente difensiva, per cui un poeta satirico di Venosa è armato, ma userà l'arma della satira solo come deterrente in grado di scoraggiare eventuali aggressioni altrui:

Hor. serm. 2, 1, 39-46

sed hic stilus haud petet ultro

quemquam animantem et me veluti custodiet ensis

vagina tectus: quem cur destringere coner 
tutus ab infestis latronibus? o pater et rex

Iuppiter, ut pereat positum robigine telum

nec quisquam noceat cupido mihi pacis! at ille,

qui me conmorit - melius non tangere, clamo -

flebit et insignis tota cantabitur urbe.

"Ma questo mio stilo mai attaccherà per primo anima viva e mi proteggerà come una spada chiusa nel fodero; perché dovrei mettermi a sguainarla, finché sono al sicuro dall'attacco dei briganti? O Giove padre e re, fa' che quest'arma, riposta com'è, sia consumata dalla ruggine e che nessuno faccia del male a me che non cerco che pace! Ma colui che mi avrà provocato (meglio non toccarmi, lo dico chiaro!) avrà di che piangere e diventerà la favola di cui si chiacchiera in tutta quanta la città. "

Credo sia giusto domandarsi, a questo punto, se l'accezione intimistica sia, in questo contesto, l'unica possibile per l'autobiografismo di Lucilio. Il filo logico dell'argomentazione suggerisce altre possibilità ${ }^{28}$. La satira è un genere che somiglia al suo poeta e la sua fisionomia dipende in larga misura dalla fisionomia della vita del satirico. I libri non sono gli unici sodales con cui Lucilio a che fare: il rapporto con i libri testimonia anzi una importante rete di sodalitas in cui la sua vita è immersa e con cui si intrecciano i singoli momenti, gli accidenti, gli umori, i pensieri. Mi sono altrove più ampiamente soffermato sul fatto che la satira è in Lucilio come "una funzione interna alla società dei pares. Il suo luogo è lo spazio pubblico, la scena della civitas, in cui i membri della classe dirigente, i primores e i loro partners, si misurano in una competizione continua, che contrappone individui e gruppi" ${ }^{29}$. La virtus, in un importante frammento di Lucilio, viene definita fra l'altro:

Lucil. 1350-2 Kr.

hostem esse atque inimicum hominum morumque malorum,

contra defensorem hominum morumque bonorum,

hos magni facere, his bene uelle, his uiuere amicum

"essere nemico e avversario di uomini e costumi malvagi, essere al contrario

difensore di uomini e costumi buoni, a questi dare stima ed affetto, con questi

vivere in amicizia"

Questa affermazione non è priva di valore programmatico: la vita stessa di Lucilio si intreccia con uomini e costumi buoni, uomini e costumi cattivi, comporta continue occasioni di consenso e dissenso, lode e biasimo, solidarietà e conflitti, successi e insuccessi. Affidare ai libri questa vita significa dunque per ciò stesso aggredire i nemici ed esaltare gli amici: per questo la satira poteva costituire un modello di poesia che ospita (secondo l'indicazione di Trebazio) l'esaltazione delle virtù di Scipione, ma certo anche l'avversione e la critica per uomini riprovevoli e ridicoli. Il focus sull'autobiografia non è dunque un modo di cambiare le carte in tavola, ma probabilmente una messa a punto delle modalità specifiche del discorso satirico. Se la satira somiglia al suo poeta, nella fisionomia biografica dei satirici è iscritta anche la diversa fisionomia del genere, a cominciare dalla declinazione del gesto fondante, quello dell'aggressione satirica.

16 Una volta stabilito che l'accento sull'autobiografia non è una via d'uscita dall'aggressività e dai problemi che essa pone, si torna perciò alla questione di fondo. E' giustificato l'allarme sociale generato dalla satira? Quali garanzie il satirico è in grado di dare? Di fronte alla congenita (e quindi irrinunciabile) predisposizione dell'ingenium del poeta verso l'uso aggressivo delle risorse che la natura stessa ha messo a sua disposizione (come le zanne per il lupo, le corna per il toro) l'allarme sociale si esprime, per bocca del giurista, in una non tanto velata minaccia: 
Hor. serm. 2, 1, 60-2

'o puer, ut sis

vitalis metuo et maiorum nequis amicus

frigore te feriat.'

"'Ragazzo mio, temo proprio che tu non abbia vita lunga e che qualcuno dei grandi,

che oggi ti è amico, non abbia a ferirti con la sua freddezza.""

Da una parte, dunque, la satira esporrebbe addirittura il suo poeta al rischio della vita, dall'altra al pericolo di perdere il favore dei grandi personaggi che gli assicurano la loro amicizia; eventualità da collegare logicamente in ordine inverso: è la perdita della solidarietà e della protezione degli amici che isola socialmente il satirico, esponendolo alla repressione più radicale, a misure estreme come quelle comminate dalla giurisdizione contro i mala carmina. Il meccanismo che la satira rischia di mettere in moto è sempre lo stesso, quello del sospetto e della paura che l'attacco satirico possa farsi indiscriminato, possa rivolgersi contro chiunque e che quindi possa verificarsi una reazione di rigetto generalizzata, ben al di là di coloro contro cui l'aggressione è stata diretta.

Di fronte a questo rischio ricorrente, probabilmente ineliminabile, la satira può offrire soltanto una garanzia, che - circolarmente e quasi tautologicamente - risiede nella vita stessa del poeta: è il fatto che i maiores sono suoi amici, che vivono con lui in rilassata intimità, al riparo da tensioni e sospetti. Se gli amici non hanno paura del satirico, se lo ammettono nella propria casa e nella propria confidenza, essi si fanno, con ciò stesso, testimoni e mallevadori contro il pericolo che il iocus degeneri in aperta rabies e che possa per honestas ire domos inpune minax (per esprimersi nei termini che il poeta dell' Epistola ad Augusto avrebbe poi applicato alla preistoria della poesia latina)

Il caso di Lucilio è particolarmente utile perché è un 'caso chiuso', e dunque dimostra storicamente la possibilità che la satira rispetti dei limiti e non si trasformi in rabies indiscriminata. L'aggressività di Lucilio aveva brutalmente smascherato i corrotti, "aveva messo sotto accusa i primi cittadini e il popolo tribù per tribù", eppure Lelio e Scipione non si erano sentiti offesi da questo suo talento polemico né avevano manifestato dolore per le lesioni inflitte a un Metello o a un Lupo:

Hor. serm. 2, 1, 65-8 num Laelius aut qui

duxit ab oppressa meritum Karthagine nomen

ingenio offensi aut laeso doluere Metello

famosisque Lupo cooperto versibus?

"forse che Lelio o colui che si meritò di trarre il soprannome da Cartagine vinta si sentirono offesi da questo suo talento o si dolsero per gli attacchi a Metello o perché Lupo veniva coperto di versi infamanti? "

Qualcuno ha osservato che ci aspetteremmo qui piuttosto la menzione di amici di Scipione attaccati da Lucilio senza che ciò ne suscitasse il risentimento ${ }^{30}$, mentre Quinto Cecilio Metello Macedonico e Lucio Cornelio Lupo erano noti piuttosto come avversari politici dello stesso Scipione. Ma l'incongruenza logica è solo apparente e si spiega bene attraverso quello slittamento dalla paura delle vittime alla paura di tutti, con cui il satirico è continuamente chiamato a fare i conti (condicione super communi, si dirà nell'Epistola ad Augusto). Scipione e Lelio, convictores di Lucilio, erano protagonisti di una ricca aneddotica che testimoniava un ininterrotto rapporto di ilare e serena intimità ${ }^{31}$ :

Hor. serm. 2, 1, 71-4

quin ubi se a volgo et scaena in secreta remorant

virtus Scipiadae et mitis sapientia Laeli 
nugari cum illo et discincti ludere, donec

decoqueretur holus soliti.

"E anzi, appena ritiratisi dalla folla e dalla scena pubblica in appartata intimità, il valoroso Scipione e Lelio, saggio e amabile, solevano scherzare con lui e giocare con la tunica discinta, in attesa che finissero di cuocere i legumi. "

Il dossier che poteva confermare questa 'garanzia' era confermato da testimonianze coeve. I lettori di Orazio potevano facilmente immaginare, se non altro grazie a Crasso, Scevola e Cicerone, il contesto di questo quasi fanciullesco relax amicale:

Cic. de orat. 2, 22

Saepe ex socero meo audivi, cum is diceret socerum suum Laelium semper fere cum Scipione solitum rusticari eosque incredibiliter repuerascere esse solitos, cum rus ex urbe tamquam e vinclis evolavissent. Non audeo dicere de talibus viris, sed tamen ita solet narrare Scaevola, conchas eos et umbilicos ad Caietam et ad Laurentum legere consuesse et ad omnem animi remissionem ludumque descendere.

"Ho sentito spesso da mio suocero, quando lui diceva che suo suocero Lelio aveva l'abitudine di villeggiare quasi sempre con Scipione e che essi erano soliti ritornare incredibilmente bambini, quando erano scappati via in campagna dalla città come da un carcere. Io non oserei parlare di tali personaggi, ma tuttavia è Scevola che suole raccontarlo, che essi avevano l'abitudine di raccogliere gusci e conchiglie sulla spiaggia di Gaeta e di Laurento e di abbandonarsi a ogni sorta di relax e di divertimento."

Ma le testimonianze più ricche si trovavano probabilmente nelle Satire stesse di Lucilio, se da lì vengono aneddoti come quello di Scipione inseguito e scherzosamente minacciato col tovagliolo, riferito (in forma leggermente diversa) dai commentatori di Orazio:

Ps. Acr. ad Hor. serm. 2, 1,72

Scipio Africanus fertur intra domum tam civilis fuisse et carus Lucilio, ut quodam tempore Laelius circa lectos triclinii fugienti supervenerit, cum eum Lucilius obtorta mappa quasi feriturus sequeretur.

"Scipione Africano si dice sia stato dentro casa sua così alla mano e così in intimità con Lucilio, che una volta Lelio lo sorprese, arrivando, che fuggiva attorno ai letti del triclinio, mentre Lucilio lo inseguiva come per colpirlo con un tovagliolo attorcigliato. " 32

A differenza di quello di Lucilio, il caso di Orazio poeta satirico è ancora 'aperto', ma l'ampiezza di testimonianze autobiografiche che le Satire oraziane stesse vanno raccogliendo costituisce un dossier non meno cospicuo e può valere come prova che, pur con le dovute differenze (infra Lucili censum ingeniumque), quando ci sarà da fare un bilancio, risulterà che Orazio ha trovato, come poeta satirico, dei garanti non meno affidabili (il dente che affonda inutilmente nella carne non sarà il suo dente di satirico, ma quello dei detrattori della satira):

Hor. serm. 2, 1, 74-78 quidquid sum ego, quamvis

infra Lucili censum ingeniumque, tamen me

cum magnis vixisse invita fatebitur usque

invidia et fragili quaerens inlidere dentem

offendet solido...

"Qualunque cosa io sia, sebbene inferiore a Lucilio per censo ed ingegno, l'invidia dovrà pur ammettere, contro voglia magari, che in ogni tempo io ho passato la vita in compagnia dei potenti e, cercando di conficcare i suoi denti nel molle, troverà il duro invece..."

La citazione della Legge delle Dodici Tavole e delle severe punizioni che (come insegnerà il poeta dell'Epistola ad Augusto) la società, per difendere il proprio assetto e la 
propria stabilità, 'opportunamente' commina contro i carmina diffamatori suona, in conclusione, come uno scrupolo di giurista più che come una minacciosa prospettiva. Del resto, il dossier autobiografico di Orazio comprende, fra i suoi 'garanti' niente meno che l'amico più grande di tutti, il principe. La frequentazione dei grandi non è del resto una realtà per cui c'è solo da fidarsi della votiva tabella tracciata da satirico stesso. Orazio presenta la città tutta e i cittadini come (invidiosi) testimoni del rapporto con Mecenate e con gli altri grandi uomini:

Hor. serm. 2, 6, 48-52 ludos spectaverat, una luserat in campo: 'fortunae filius' omnes. frigidus a rostris manat per compita rumor: quicumque obvius est, me consulit: 'o bone - nam te scire, deos quoniam propius contingis oportet -

...

"aveva assistito ai giochi insieme a lui? con lui aveva giocato nel Campo? E tutti: 'Eccolo il figlio della fortuna!'. Una gelida voce dai Rostri, attraverso i crocicchi si spande; chiunque m'incontri, mi prende a consulto: 'Esimio, tu non puoi non saperlo, visto che vivi a stretto contatto con gli dèi..."

Che la scrittura autobiografica abbia un legame organico con l'intenzione apologetica è stato più volte osservato: scrivono di sé soprattutto personaggi che sentono criticata la propria condotta o messa in discussione la propria immagine. In un certo senso si può dire che questo è vero anche per i generi letterari e in particolare per la satira. Il satirico costruisce la sua persona con materiali autobiografici per accreditare come un prodotto socialmente accettabile questo pericoloso genere letterario latino.

\section{BIBLIOGRAFIA}

Barchiesi 1993: A. Barchiesi, «Insegnare ad Augusto. Orazio, Epistole 2, 1 e Ovidio Tristia II», "MD" 31, 1993, 149-84.

Barchiesi-Cucchiarelli 2005: A. Barchiesi, A. Cucchiarelli, «Satire and the poet: the body as selfreferential symbol», K. Freudenburg (ed.), The Cambridge Companion to Roman Satire, Cambridge 2005, 207-223.

Brink 1971: C. O. Brink, Horace on Poetry. The 'Ars Poetica', Cambridge 1971.

Brink 1982: C. O. Brink, Horace on Poetry. Epistles Book II: the Letters to Augustus and Florus, Cambridge 1982.

Canobbio 2016: A. Canobbio, «Lucilius and Horace: from criticism to identification», in Papers of the Langford Latin Seminar, vol. 16, 2016: Greek and Roman Poetry; The Elder Pliny, ed. by F. Cairns , R. Gibson, Prenton 2016, pp. 185-205.

Citroni 1991: M. Citroni, «L'autobiografia nella satira e nell'epigramma latino», in G. Arrighetti, F. Montanari (a cura di), La componente autobiografica nella poesia greca e latina fra realtà e artificio letterario, Pisa 1991, 275-92. 
Citroni 2013: M. Citroni, «Horace's Epistle 2. 1, Cicero, Varro and the Ancient Debate about the Origins and the Development of Latin Poetry», in J. Farrell, D. P. Nelis (eds.), Augustan Poetry and the Roman Republic, Oxford 2013, 180-204.

Citroni Marchetti 2004: S. Citroni Marchetti, «I precetti paterni e le lezioni dei filosofi: Demea, il padre di Orazio,e altri padri e figli», "MD" 53, 2004, 9-63.

Conte 1966: G. B. Conte, «Uno studioso tedesco di letteratura latina: F. Klingner», "Critica storica" 1966, 481-503.

Conte 1984: G. B. Conte, Virgilio. Il genere e i suoi confini, Milano 1984.

Conte 1986: G. B. Conte, The Rhetoric of Imitation: Genre and Poetic Memory in Vergil and Other Latin Poets, Ithaca \& London 1986.

Cucchiarelli 2001: A. Cucchiarelli, La satira e il poeta. Orazio tra Epodi e Sermones, Pisa 2001.

Delignon 2006, B. Delignon, Les Satires d'Horace et la comédie gréco-latine: une poétique de l'ambiguité, Louvain- Paris-Dudley 2006.

Du Quesnay 1984: I. M. LeM. DuQuesnay, «Horace and Maecenas: The Propaganda Value of Sermones 1», in A. J. Woodman, D. West (eds.), Poetry and Politics in the Age of Augustus, Cambridge 1984, 19-58 = K. Freudenburg (ed.), Oxford Readings in Classical Studies. Horace, Satires and Epistles, Oxford 2009, 42-101.

Fedeli 1994: P. Fedeli, Q. Orazio Flacco, Le Opere, II.2, Le Satire, Roma 1994.

Freudenburg 1993: K. Freudenburg, The Walking Muse: Horace on the Theory of Satire, Princeton, 1993.

Gelli 2014: E. Gelli, «Tracce di onomastì komodèin dalla Commedia di Mezzo a Menandro», in A. Casanova (a cura di), Menandro e l'evoluzione della commedia greca, Firenze 2014, 63-81.

Gowers 2003: E. Gowers, «Fragments of Autobiography in Horace Satires 1», "Class. Ant." 22, 2003, 55-91.

Gowers 2012: E. Gowers, Horace, Satires Book I, Cambridge 2012.

Haliwell 1991: S. Haliwell, «Comic Satire and Freedom of Speech in Classical Athens», "JHS” 111, 1991, 48-70

Harrison 1987: G. Harrison, «The Confessions of Lucilius (Hor. Sat. 2.1.30-34): A Defense of Autobiographical Satire?», "Class. Ant." 6, 1987, 38-52.

Harrison 2007: S. J. Harrison, «Horatian Self-representations», in S. J. Harrison (ed.), The Cambridge Companion to Horace, Cambridge 2007, 22-35.

Harrison 2013: S. J. Harrison, «Author and speaker(s) in Horace's Satires 2», in A. Marmadoro, J. Hill (eds), The Author's Voice in Classical and Late Antiquity, Oxford 2013, 153-71.

Hendrickson 1900: G. L. Hendrickson, «Horace, Serm. 1. 4: A Protest and a Programme», "AJPh" 21, $1900,121-42$

Kiessling-Heinze 1957: A. Kiessling, R. Heinze, Q. Horatius Flaccus, Satiren, Berlin $1957^{6}$

Labate 1992: M. Labate, «Le necessità del poeta satirico: fisiopatologia di una scelta letteraria», in I. Mazzini (a cura di), Civiltà materiale e letteratura nel mondo antico, Macerata 1992, 55-66.

Labate 2012: M. Labate, «La satira e i suoi bersagli: dallo spazio della civitas allo spazio della corte», in M. Citroni (a cura di), Letteratura e civitas. Transizioni dalla Repubblica all'Impero, Pisa 2012, 269-93. 
Labate-Rosati 2013: M. Labate, G. Rosati , «'Tua, Caesar, aetas': un personaggio, un'epoca, un mito. Riflessioni preliminari», in M. Labate, G. Rosati (a cura di), La costruzione del mito augusteo, Heidelberg 2013, 1-28.

La Penna 1960: A. La Penna, Orazio, Satire ed Epistole, Firenze 1960.

Lowrie 2009: M. Lowrie, Writing, Performance and Authority in Augustan Rome, Oxford 2009.

Muecke 1979: F. Muecke, «Horace the Satirist: Form and Method in Satires 1.4», "Prudentia" 11, 1979, 55-68.

Muecke 1993: F. Muecke, Horace, Satires II, Warminster 1993.

Muecke 1995: F. Muecke, «Law, Rhetoric and Genre in Horace, Satires 2. 1», in S.J. Harrison, Homage to Horace: A Bimillenary Celebration, Oxford 1995, 203-18.

Rosenbloom 2014: D: Rosenbloom, «The Politics of Comic Athens», in M. Fontaine, A. C. Scafuro (eds), The Oxford Handbook of Greek and Roman Comedy, Oxford 2014, 297-320.

Tatum 1998: W. J. Tatum, «Ultra legem: Law and Literature in Horace's Satires II 1», "Mnemosyne" $51,1988,688-99$.

Waszink 1948: J. H. Waszink, «Varro, Livy and Tertullian», "Vigiliae Christianae" 11, 1948, 221-42.

Zetzel 1980: J. E. G. Zetzel, «Horace's Liber Sermonum: The Structure of Ambiguity», “Arethusa” 13, 1980, 59-77 = K. Freudenburg (ed.), Oxford Readings in Classical Studies. Horace, Satires and Epistles, Oxford 2009, 17-41.

\section{NOTE}

1. La questione era notoriamente connessa al sorgere delle forme primitive di performance drammatica: un quadro sintetico delle principali testimonianze antiche e della loro relazione con la teoria (o le teorie) varroniane, con una discussione della precedente bibliografia, in Brink 1982, 179-86 (in particolare sulla posizione di Orazio 183-6).

2. Per una convincente discussione del testo, dove la congettura invecta, attribuita a Poliziano, si fa preferire alla lezione tràdita inventa, vedi Brink 1982, 192.

3. La connessione tra antichissime forme ludico-drammatiche, Fescennina licentia e rusticitas dei versus incompti, comune nelle testimonianze augustee, risale forse all'autorità di Varrone: Waszink 1948, 241; nella stessa direzione, ma più prudente, Brink 1982, 182.

4. L'espressione ha probabilmente valenza generale: la preoccupazione sulla sorte che può accomunare tutti si traduce in una preoccupazione di ordine politico-sociale: "state or order of the body politic" (Brink 1982, 195).

5. Il riferimento più probabile è alla Legge delle XII Tavole: cfr. Brink 1982, 196.

6. Brink 1982, 184.

7. Cfr. Rosenbloom 2014, con bibliografia precedente. Nella cosiddetta 'commedia di mezzo' il processo è complesso, graduale: cfr. Gelli 2014. Sul ruolo discusso della legislazione ateniese contro la diffamazione, Brink 1971, 316-7; Haliwell 1991, 49-50; Gelli 2014, 63-4, con ulteriore bibliografia.

8. Barchiesi 1993. L'Epistola ad Augusto è stata oggetto di notevoli analisi di studiosi oraziani di primissimo piano: ottimo il contributo recente di Citroni 2013.

9. Labate-Rosati 2013, 13-17.

10. Un anonimo referee della rivista mi segnala giustamente in Hor. serm. 1, 3, 99-112 un altro sguardo retrospettivo, che collega in maniera implicita l'evoluzione, lucrezianamente delineata, dalla violenza atavica alla civiltà con l'evoluzione del genere satirico e l'integrazione sociale del 
poeta satirico capace di indulgenza e di rinuncia all'aggressività indiscriminata: si veda Gowers 2003, 75 e Gowers 2012, 137-8.

11. Si tratta di un aspetto molto presente negli studi oraziani. Ricordo soltanto il pregevole contributo recente di Delignon 2006, con ampia bibliografia. Vd. ora anche Canobbio 2016, 185-205.

12. Vd. soprattutto Du Quesnay 1984 (=2009, 54-62) Freudenburg 1993, 86-92.

13. Cfr. Muecke 1979, 57-9.

14. Hendrickson 1900; Freudenburg 1993, 52 ss., Muecke 1979, 62 ss.

15. Per la questione testuale gli elementi essenziali in Gowers 2012, 161.

16. La discussione sugli elementi autobiografici e la funzione dell'autobiografia è variamente connessa agli studi sul concetto di 'persona' nella letteratura antica (e satirica in particolare), che, partire da Kernan 1949 si sono fatti strada negli studi di letteratura latina soprattutto attraverso i lavori confluiti in Anderson 1982, fino a risultare assolutamente dominanti nel panorama critico di più di tre decenni: ricordo qui soltanto, tra i contributi più specifici per il nostro tema, Harrison 1987 e Gowers 2003. Da vedere comunque Zetzel 1980, 60-1, Muecke 2007, 106-9; Harrison 2007, e tra le voci (moderatamente) critiche Citroni 1991 e Mayer 2003.

17. Convincenti argomentazioni in Kiessling-Heinze $1957^{6}$ 80-1 e Gowers 2012, 171-2.

18. Del ruolo fondamentale della satira 1, 3 nella definizione oraziana dell'aggressività del satirico mi sono occupato in Labate 2012, spec. 288 ss.

19. Sull'attribuzione della battuta e il rapporto con 1, 6, 51 ss, vedi ora anche Gowers 2012, 294 .

20. Cfr. Citroni Marchetti 2004, 19 ss.

21. Uno dei tentativi più convincenti è Muecke 1995: cfr. Labate 2012, 283 ss. con riferimenti bibliografici. Da vedere anche Tatum 1998 e Lowrie 2009, 327-48.

22. Conte 1984, 14 = Conte 1986, 101-2. Vedi anche Conte 1966.

23. La maggior parte dei commentatori insistono in maniera secondo me unilaterale sull'accezione stilistico-retorica di sine nervis (Cfr. Kiessling-Heinze 19576 , 179; Muecke 1993, 101). Più equilibrato Fedeli 1994, 532-3.

24. Labate 1992. Da vedere Cucchiarelli 2001, 66 ss. e Barchiesi- Cucchiarelli 2005.

25. Cfr. Kiessling-Heinze $1957^{6}, 182$.

26. Cfr. Labate 2012, 285-6.

27. Mi paiono fuori strada coloro che (Kiessling-Heinze $1957^{6}, 184$; Muecke 1993), per eccesso di 'precisione' autobiografica, intendono che "Horace claims descent not from the farmer-colonists but from one of their warlike neighbours, some of whom were no doubt captured and enslaved". Non bisogna mai dimenticare gli elementi autobiografici sono funzionali alla persona satirica che il poeta intende costruire.

28. Un'interpretazione diversa dalla mia, ma altrettanto attenta a queste possibilità, è quella di Harrison 1987.

29. Labate 2012, 270.

30. La Penna 1960, 118

31. Cfr. Cucchiarelli 2001, 73.

32. In forma leggeremente diversa (e alquanto più oscura), l'aneddoto è riferito dagli Schol. Cruq. ad Hor. serm. 2, 1, 72: Scipio Africanus et Laelius feruntur tam fuisse familiares et amici Lucilio, ut quodam tempore Laelio circum lectos triclinii fugienti Lucilius superveniens cum obtorta mappa quasi feriturus sequeretur. 


\section{RIASSUNTI}

L'articolo propone una nuova discussione dell'autobiografia come elemento chiave della satira luciliana e oraziana: si insiste sulla caratterizzazione del genere attraverso la fisionomia dell'autore e dettagli personali a lui pertinenti, nonché sul ruolo dell'amicizia con i grandi come garanzia dell'innocuità e della controllabilità sociale di un genere poetico costituzionalmente destinato a suscitare diffidenza, allarme, rigetto. L'analisi parte dalla quella specie di archeologia del genere prospettata nell'Epistola 2,1 ed è condotta attraverso una discussione di luoghi programmatici delle Satire: soprattutto 1,4 e 2, 1, di cui è proposta un'analisi ravvicinata, che cerca di ricostruire il difficile filo logico che si dipana nel componimento nel nesso non sempre perspicuo tra aggressività e autobiografia.

\section{INDICE}

Mots-clés : Satira, Autobiografia, Aggressività, Orazio, Lucilio 